\title{
Plan lector en inglés apoyado en revistas digitales para cursos disciplinares del programa de Ingeniería Civil, utilizando la metodología ESP
}

\author{
Wilson Enrique Torres Sánchez, M.Sc ${ }^{1}$, Mary Elen Niño Molina, M.Sc ${ }^{1}$, \\ ${ }^{1}$ Universidad la Gran Colombia, Colombia, wilson.torres@ugc.edu.co, mary.nino@ugc.edu.co
}

\begin{abstract}
Resumen- Este artículo de investigación describe la importancia del idioma inglés como lengua extranjera dentro de un proceso de indagación, gamificación, aprendizaje autónomo y lectura digital. El objetivo planteado era desarrollar una propuesta digital que potenciara el uso del inglés técnico aplicado al plan lector en cuatro cursos del programa de Ingeniería Civil mediante un entorno virtual basado en revistas interactivas propias de la disciplina y apoyadas en la metodología ESP (inglés para propósitos específicos), enmarcado dentro del plan lector de los syllabus de los cuatro cursos. La situación de cuarentena obligatoria generada por el Covid-19 hizo que parte del proceso metodológico y el trabajo de campo fueran $100 \%$ virtuales. La investigación se realizó mediante una metodológica cuantitativa correlacional y la muestra intervenida fue de 161 estudiantes. Dentro de los resultados obtenidos se destaca que el plan lector planteado a partir de las revistas digitales potenció el nivel de inglés de los estudiantes, de acuerdo con los parámetros estadísticos utilizados y el tipo de investigación. Se aprovechó la situación de educación virtualizada para fortalecer las temáticas disciplinares, propias del ingeniero civil y las habilidades de la lengua extranjera.

Palabras clave-- Plan lector, Inglés para Propósitos Específicos (ESP), Revistas Digitales, Entornos Virtuales de Aprendizaje.
\end{abstract}

Abstract-This research article describes the importance of the English language as a foreign language within a inquiry, gamification, autonomous learning and digital reading process. The main objective was about developing a digital proposal that would promote the use of technical English applied to the reading plan in some courses of the civil engineering program through a virtual environment, it was based on interactive and digital magazines. They worked about the civil engineering discipline and they were focused on the ESP methodology. It was framed within the reading plan established through the syllabus of the four disciplinary courses. The mandatory quarantine situation generated by Covid-19 made part of the methodological process and fieldwork research. Just because it was $100 \%$ virtual. The research was carried out using a correlational quantitative methodological. The intervened sample was 161 students. Talking about the results obtained, it is highlighted that the reading plan proposed from the digital magazines enhanced the level of English to the civil engineering students, it was according to statistical facts used to measure different aspects from the type of research selected. The situation of virtualized education was used to strengthen disciplinary themes about civil engineering program and foreign language skills.

Keywords-- Reading plan, English for Specific Purposes (ESP) digital magazines, virtual learning Environment.

Digital Object Identifier (DOI):

http://dx.doi.org/10.18687/LEIRD2021.1.1.49

ISSN: 2414-6390 ISBN: 978-958-52071-9-6

\section{INTRODUCCIÓN}

En la primera parte del artículo se establecieron los antecedentes, las teorías y procesos pedagógicos con el objetivo de mejorar los niveles de aprehensión del idioma inglés a partir del plan lector establecido en cuatro cursos disciplinares del programa de Ingeniería Civil de la Universidad la Gran Colombia, enmarcados dentro la importancia del inglés como lengua extranjera (English as a Foreign Language, EFL) y apoyados en la metodología ESP (English for specific Purposes, en español Inglés para propósitos Específicos). Enseguida, se abordarán los aspectos metodológicos que permitieron crear entornos virtuales apoyados en revistas digitales. Más adelante, se describirá el proceso de validación de las revistas con la intervención de 161 estudiantes que conformaron la muestra. Los resultados permitirán analizar la intervención de acuerdo al tipo de investigación escogido. Finalmente, las conclusiones contrastarán la pregunta de investigación, los objetivos y algunos teóricos que soportaron el proceso. Cabe anotar que parte de la construcción de las revistas y todo el trabajo de campo se realizó de manera virtual y, aunque los cursos seleccionados eran presenciales, la situación de cuarentena obligatoria generada por el Covid-19 obligó a que la implementación del plan lector apoyado en las revistas fuera $100 \%$ virtual.

1.1 Las estrategias pedagógicas y tecnológicas en el aprendizaje del idioma inglés

[9] Thomas y Reinders (2010), en su libro Task based language learning and teaching with tecnology, plantea el uso de la tecnología y el enfoque TBLT (Task Based Learning Teaching) como una estrategia para enfrentar dos grandes paradigmas que tiene la enseñanza-aprendizaje del lenguaje como son el enfoque psicolingüista y el enfoque sociocultural, permitiendo que el aprendizaje de una segunda lengua mediado por la tecnología incorpore situaciones de la vida real (cotidiana) y de igual manera los contextos socioculturales de los estudiantes, favoreciendo en primer lugar el desarrollo de las habilidades comunicativas en Inglés y en segundo lugar a la motivación y trabajo cooperativo de los mismos. Además de brindar descripciones muy específicas de los roles de cada uno de los actores en este proceso tales como el rol del docente y estudiante, también profundizan en conceptos importantes a la hora de hablar de tecnología como herramienta facilitadora del aprendizaje como son TB-SCMC (Task based Synchronous 
Computer Mediated Communication) y VLE (Virtual Learning Environment), los cuales enfocan la atención de los estudiantes en aspectos formales del lenguaje, permitiéndoles cuestionarse del uso del mismo en diferentes escenarios y con el apoyo constante de la tecnología para que dichos escenarios logren ser lo más reales y significativos posibles.

Por otra parte, el trabajo autónomo es un aspecto fundamental para el dominio de la lengua extranjera, como señala la investigación de [7] Gary Murray y Terry Lamb (2011, p. 177) la mayoría de estudiantes que desarrollan su proceso de aprendizaje del Inglés como lengua extranjera, se encuentran en una continua lucha que requiere un nivel más alto de autonomía y motivación, con el análisis realizado, se pudo determinar que los estudiantes que estaban más motivados y dedicaban más tiempo en su trabajo autónomo tenían un mejor dominio del idioma que no les requería cambiar o "code-switching" a sus lenguas maternas para comunicarse. Con las entrevistas llevadas a cabo en la investigación, se determinó que estos estudiantes dedicaban más tiempo a su proceso autónomo debido a que tuvieron mejores herramientas para usar fuera del aula lo cual los motivó a continuar su proceso de aprendizaje.

Estos autores también señalan que el trabajo autónomo es parte fundamental en el aprendizaje de un segundo idioma, es por ello que desarrollaron un análisis el cual determinó que los estudiantes de EFL que tienen mayor motivación y dedican más tiempo a su aprendizaje incluyendo actividades de trabajo autónomo, tenían un mejor desempeño en el idioma tanto en habilidades receptivas como en las habilidades productivas, permitiendo que los estudiantes desarrollen las temáticas con actividades basadas en los intereses de cada uno, tales como: las artes, la ciencia, la ingeniería, la historia, entre otros, generando estímulos positivos en el proceso de aprendizaje y permitiéndoles disfrutar y expandir su conocimiento en distintas áreas, además de utilizar el Inglés como una herramienta mediadora de comunicación. Asimismo, enfatizan la importancia de las nuevas metodologías de enseñanza del inglés, las cuales centran su diseño en el estudiante, donde este tiene un rol cada día más activo para gestionar su proceso y desarrollo del aprendizaje.

En ese sentido, uno de los problemas más comunes de los docentes de EFL es la falta de motivación de los estudiantes; con la investigación llevada por [8] Boukhatem Nadera en Turquía (2015) con la educación mediada por ayudas digitales, los estudiantes realizaban las actividades y juegos de una manera dinámica y entretenida, y son una excelente herramienta de aprendizaje para enseñar vocabulario y sintaxis, de igual manera determinó que uno de los mejores aspectos de utilizar juegos y situaciones digitales, es que son agradables y transfieren las palabras a eventos diferentes y maximizan su alcance, por lo que el entorno virtual se transforma en una herramienta que elimina el miedo, la ansiedad y el aburrimiento en las clases, factores que influyen en gran medida a la motivación de los estudiantes para aprender una lengua extranjera, y al ser una herramienta de fácil acceso, contribuye a su desarrollo de aprendizaje autónomo, en la medida en que los estudiantes pueden utilizar otro tipo de actividades y potenciar sus necesidades específicas frente a temáticas propias de su profesión.

De igual forma, es importante resaltar el proyecto de investigación de [5] Lina Lee de la Universidad de New Hampshire (2016) titulado "Autonomous Learning Through Task-Based Instruction In Fully Online Language Courses", en el que expone, según los resultados obtenidos de 48 estudiantes, como las herramientas tecnológicas y el uso de TBI (TaskBased Instruction) les permite a los estudiantes tener un rol más autónomo durante su proceso de aprendizaje y del mismo modo los mantenía motivados mientras podían trabajar por su cuenta o con la ayuda de sus compañeros. Con respecto al uso de herramientas tecnológicas, [5] Lee afirma que los estudiantes las veían como una manera cómoda y fácil de trabajar, por lo que se convertían de su agrado. Aunque el proyecto está enfocado a proceso de aprendizaje de las cuatro habilidades completamente online, las conclusiones y recomendaciones encontradas reafirman el uso de herramientas digitales como sitios web y el uso de una metodología TBI para motivar a los estudiantes y al mismo modo ir generando y desarrollando autonomía frente a su proceso de aprendizaje para que con las instrucciones y la retroalimentación del docente, los estudiantes entiendan de qué manera pueden ir tomando un rol más activo frente a su desempeño y dominio de la lengua extranjera.

En Colombia se han buscado diferentes formas de enseñar una lengua extranjera, esto con el propósito de motivar, justificar y promover el aprendizaje de manera variada:

"profesionales de la educación y la industria por igual están obligados a repensar el papel de los sistemas educativos de sus países, en busca de nuevos enfoques para preparar a los estudiantes para tener éxito en una sociedad cada vez más basada en la información y la economía que prevalece tanto en contextos locales y globales" [6] (McDougald, 2014, p. 3).

En el marco de estas formas se destaca CLIL (por sus siglas en inglés Content Language and Integrated Learning). "La introducción de un enfoque de Aprendizaje Integrado de Contenidos y Lenguas Extranjeras AICLE en el sistema educativo general de Colombia es una opción viable para muchos" [6] (McDougald, 2014, p. 33). En español, la terminología cambia, ya no es CLIL, sino que se le conoce mejor como AICLE "es un enfoque educativo con un objetivo doble en el que un idioma adicional se utiliza para la enseñanza y el aprendizaje de contenidos y lengua" [6] (Coyle, Hood, y Marsh, 2010 citados en McDougald, 2014, p. 34). En todo caso, la idea es la misma. Así las cosas, el presente proyecto de investigación se enmarca en esa función de enseñar de una manera alterna y, principalmente, que tenga un doble propósito: 
aprender inglés y aprender contenido, en este caso, atado a la Ingeniería Civil.

De otro lado, surge otra forma de aprender que se ajusta mejor al presente proyecto y es la metodología ESP (English for specific Purposes), la cual se traduce como inglés para fines específicos, y del cual se desprenden dos ramas: inglés para fines ocupacionales e inglés para fines académicos. De acuerdo con [10] Vidal (2005), la distinción entre ambas ramas parece no ser muy clara, ya que una rama ha dominado a la otra, la académica. Sin embargo, [3] Bueno y Hernández (2012) hacen una clasificación de esas dos ramas que surgen a partir del inglés con fines específicos y que se observa en la figura 1:

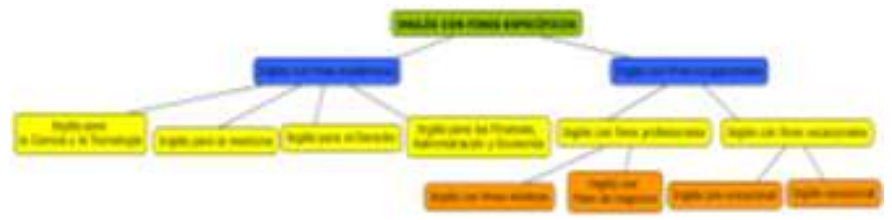

Figura 1. Clasificación de IFE por área profesional realizada por Bueno y Hernández (2012)

Para el caso de la presente investigación, la rama que mejor se ajusta es el inglés con fines académicos, ya que se hace en un contexto educativo, con una base de aprendizaje de ingeniería civil y se fortalece el inglés a través de las temáticas propias del plan educativo profesional.

Esta metodología se adapta a las diferentes formas de aprendizaje, es el caso del blended learning que destaca la combinación de dos métodos de instrucción, en primer lugar, la educación a distancia con el apoyo de herramientas digitales y en segundo lugar la instrucción presencial o como es conocida coloquialmente instrucción cara a cara, esto con el fin de lograr un óptimo desarrollo en el proceso de enseñanza-aprendizaje. Por su parte, [2] Bonk, C. J., \& Graham, C. R. (2012), destacan los beneficios de este enfoque metodológico y la combinación de múltiples instrucciones en el aprendizaje del siglo XXl en su libro "The handbook of blended learning", en donde también enfatizan el uso de recursos tecnológicos como instrumentos innovadores y necesarios para afrontar los retos de la educación actual, además de resaltar la importancia de la instrucción y el monitoreo presencial por parte del docente, teniendo en cuenta las diferentes perspectivas, modelos y categorías del blended learning systems. De esta manera, este enfoque metodológico complementa bien el objetivo principal de esta investigación, debido a que incorpora las propiedades tales como el uso de la tecnología en pro a la educación y el método de enseñanza virtual o semipresencial en donde el docente es el encargado de observar y guiar el proceso pedagógico ya sea a través de la virtualidad o su acceso al aula.

Por otra parte, La educación superior está viviendo un momento caracterizado por la globalización, lo que supone en países como Colombia un perfil y un accionar exigente $\mathrm{y}$ competitivo. Ante estas demandas el docente universitario debe dar cara a cada desafío, independiente de su disciplina, lo que conlleva a estar más y mejor preparados que los estudiantes. Aquí el inglés juega un papel importante; de acuerdo al Ministerio de Educación Nacional Colombiano (MEN, 2015) el aprendizaje del idioma inglés es un proceso esencial para el desarrollo y el éxito de los estudiantes en el siglo XXI. Por tal motivo, el aprendizaje y uso competente de este idioma es una necesidad latente. Cabe señalar aquí a [1] Lave y Wenger (citado en Bhatia, 2008) quienes señalan que el principal objetivo de un acercamiento del Inglés con Fines Específicos (IFE) es la enseñanza y el aprendizaje del idioma, aunque en situaciones y contextos especiales y específicos.

Teniendo en cuenta el panorama anterior, surge la necesidad de implementar una herramienta digital que ayude a los docentes a mejorar la habilidad en inglés de sus estudiantes, haciendo uso efectivo de la enseñanza del idioma enfocada a situaciones auténticas relacionadas con la Ingeniería civil, es decir, al mundo ocupacional que a ellos les concierne. De acuerdo con este contexto, se plantea la siguiente pregunta que oriente estos procesos en la investigación: ¿Cómo potenciar el uso del inglés técnico aplicado al plan lector en unos cursos del programa de Ingeniería Civil mediante un entorno virtual basado en revistas interactivas propias de la disciplina $y$ apoyadas en la metodología ESP?

\section{METODOLOGÍA}

La población que intervino en la etapa inicial del proyecto estuvo compuesta por 20 estudiantes de programa de Licenciatura en Lenguas Modernas con énfasis en Inglés que hacían parte del curso Interdisciplinary English (Inglés interdisciplinario) del programa de Licenciatura en Lenguas Modernas con Énfasis en Inglés, y el cual se encargó de la construcción de las revistas. De igual forma, se sumaron a este equipo cinco estudiantes pasantes del programa de Ingeniería Civil, dos de ellas fueron las asesoras técnicas durante todo el proceso de construcción de las revistas, el tercero ayudó para realizar la prueba de usuario de las revistas y el posterior ajuste de las mismas, y las otras dos personas pasantes monitorearon el trabajo de campo. La población intervenida fueron los estudiantes del programa de Ingeniería Civil que hacían parte de los cursos apoyados en las revistas digitales, quienes fueron el equipo que permitió validar el trabajo con las revistas. Este último grupo en total fue de 161 estudiantes que se distribuyeron en 4 entornos virtuales, cada uno apoyado por una revista. Para seleccionar los syllabus y posteriores temáticas de los cursos, se contó con el apoyo de profesores disciplinares de los cuatro cursos específicos del programa de Ingeniería Civil.

Por otra parte, la presente investigación fue principalmente de tipo correlacional. En ese sentido, hubo la necesidad de comparar el antes y el después de los estudiantes en el proceso y contrastar los resultados, para ello se utilizó este tipo de 
investigación, el cual como dice [4] Hernández (2010) “este tipo de estudios tiene como finalidad conocer la relación o grado de asociación que exista entre dos o más conceptos, categorías o variables en una muestra o contexto en particular". De acuerdo a lo anterior se desarrolló el proyecto y permitió tomar información de la siguiente forma a partir del trabajo de campo:
a. Pretest.
b. Revisión de las revistas.
c. Asesoría de los investigadores.
d. Actividades en las revistas.
e. Postest.
f. Encuesta de percepción.

Esta estructura fue soportada con las siguientes fases:

\section{Fase 1. Indagación.}

Esta primera fase contó con la intervención de los dos investigadores y 4 profesores disciplinares del programa de ingeniería civil que se encargaron de seleccionar los syllabus disciplinares a intervenir, los cuales fueron: Materiales de Construcción, Estática, Hidráulica y Tránsito y Transporte. Enseguida a ello, Se unieron al equipo dos estudiantes pasantes del programa de Ingeniería Civil, quienes se encargaron, con orientación de los profesores y los syllabus, de hacer la base de datos de los temas, a partir de diferentes insumos y ayudas en línea para cada una de las temáticas base de las revistas.

\section{Fase 2. Diseño}

De esta fase hicieron parte los investigadores y las estudiantes pasantes, quienes reunieron las bases de datos construidas en la fase de indagación. De igual forma, se establecieron aspectos básicos de forma en la confección de las revistas, como lo fueron: la portada, los créditos, la tabla de contenido (que se estructuró desde 4 unidades), la introducción, la justificación, el cuerpo de las revistas a partir de esas cuatro unidades y Referentes bibliográficos. En la segunda parte de esta fase se unieron al equipo de trabajo 20 estudiantes del curso Interdisciplinary English de la Licenciatura en Lenguas Modernas con Énfasis en Inglés, a quienes se les dividió en grupos para trabajar en las revistas, también se les explicó la estructura de las mismas y se les dio a conocer las bases de datos. Todo lo que tenía que ver con la parte gráfica de las revistas y las actividades de gamificación debía ser creación de cada grupo con la revisión constante disciplinar de las dos estudiantes pasantes y la asesoría y revisión pedagógica y de idioma por parte de los investigadores. Con base en estos aspectos y estructura, se realizó la construcción de las revistas. En la figura 2 se observa la portada de una de ellas:

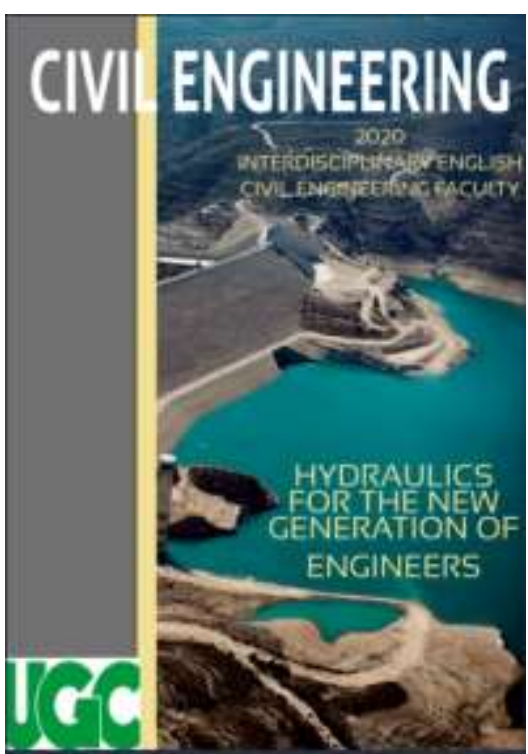

Figura 2. Portada de la revista de Hidráulica.

Cabe anotar que, a medida que se avanzaba en la construcción de las revistas, también se iban elaborando las pruebas pre $\mathrm{y}$ postest fundamentales para el tipo de investigación.

\section{Fase 3. Construcción.}

En esta fase se procedió a la elaboración de las revistas y de acuerdo con los postulados del enfoque ESP. En ese sentido, hubo una triangulación investigadores - estudiantes pasantes estudiantes de la licenciatura, que permitió la construcción de las cuatro revistas. En la figura 3 se observa la tabla de contenido y los créditos de una de las revistas:

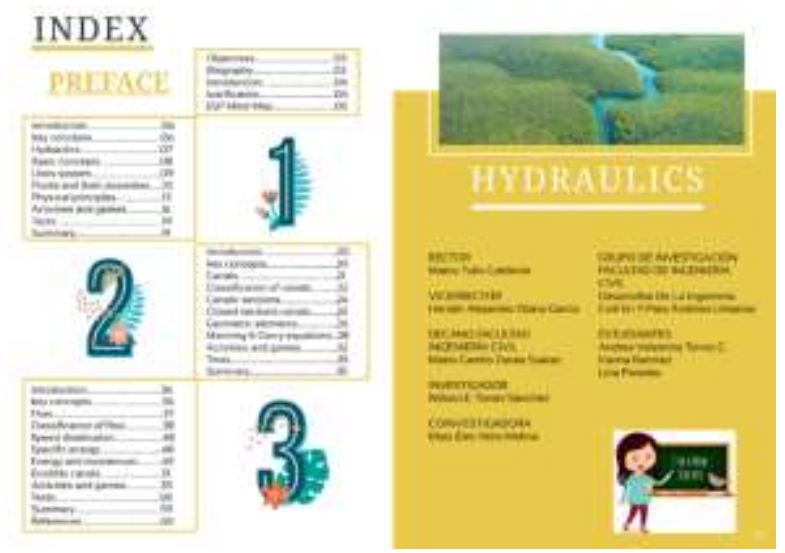

Figura 3. Tabla de contenido y créditos de la revista sobre Hidráulica.

En la segunda parte de esta fase se hizo una prueba de usuario sobre cada revista, de tal forma que se pudieron identificar aspectos de forma y de fondo que debían ajustarse, la misma la llevó a cabo el tercer estudiante pasante del programa de Ingeniería civil y a partir de un instrumento 
cualitativo que permitiera identificar os aspectos antes mencionados.

Finalmente, y una vez teniendo listas las revistas, los investigadores crearon los cuatro ambientes virtuales desde la plataforma Moodle, en donde se incluyeron también las pruebas pretest y postest. En la figura 4 se observa el espacio de uno de los entornos virtuales en donde se apoyaron las revistas

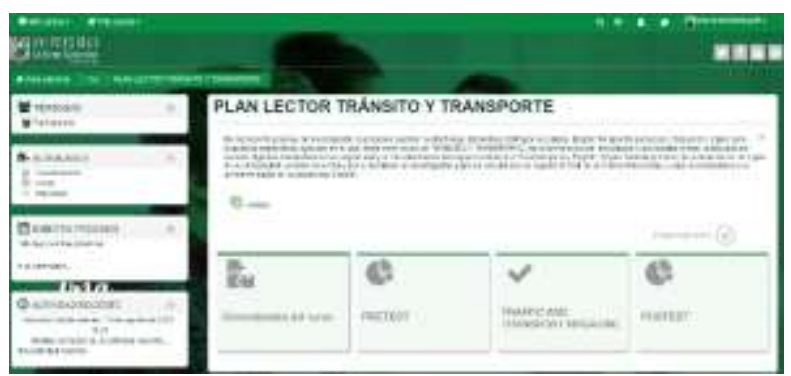

Figura 4. Ambiente virtual para la revista sobre Construcción e Interventoría.

Como se observa en la figura 4 , la estructura de cada entorno virtual se conformaba en la parte superior del título del plan lector correspondiente y una introducción, los dos en español. En la parte de abajo se observan cuatro temas: el primero da el ingreso a las generalidades del curso, luego está el tema que da el ingreso al pretest, y el cual se habilitó entes de realizar la intervención, enseguida está el tema que da el ingreso a la revista, la cual está $100 \%$ en idioma inglés, y, por último, se encuentra el postest, el cual se habilitó después de la revisión de la revista. Una vez terminado el postest por parte de los estudiantes de la muestra, se encontraban con la encuesta de percepción del proceso desarrollado. Cabe destacar que todo el proceso se llevó a cabo por cerca de 4 semanas.

\section{Fase 4. Intervención}

En esta fase se tomó una muestra no probabilística por conveniencia de 161 estudiantes que hacían parte de los cursos base de las revistas digitales. La distribución de los estudiantes que hicieron parte del trabajo de campo se presenta en la tabla 1:

\begin{tabular}{|l|l|}
\hline REVISTA & CANTIDAD \\
\hline MATERIALES DE & \\
CONSTRUCCIÓN & 56 \\
\hline ESTÁTICA & 36 \\
\hline HIDRÁULICA & 22 \\
\hline TRÁNSITO Y TRANSPORTE & 47 \\
\hline
\end{tabular}

Tabla 1. Distribución de estudiantes por entorno virtual que realizaron el proceso completo.

Como se observa en la tabla 1, la cantidad de estudiantes no fue la misma por entorno, ya que eso dependió de los que estaban tomando cada curso. Teniendo en cuenta lo anterior, en esta etapa se realizó una interacción entre las personas participantes en todo el trabajo de campo: muestra, pasantes e investigadores. También se contó con la colaboración de cada uno de los profesores titulares de los cursos intervenidos. En primera instancia, se reunieron de forma virtual a los estudiantes de los cuatro grupos. Una vez explicada la estrategia por parte de los investigadores, se procedió a mostrar cada uno de los entornos virtuales y la metodología de trabajo en los mismos. Como gran parte del éxito al trabajar en este entorno dependía de los estudiantes de ingeniería, se les orientó para desarrollar de buena forma su trabajo en dicho entorno. El trabajo de los estudiantes, en general, debería ser muy autónomo, pero se acordaron unas tutorías semanales para aclarar dudas y reforzar algunos aspectos de idioma, a partir de las dudas, si así ellos lo decidían, en esta parte contaron con la asesoría de las dos estudiantes pasantes. Sin otro particular, el trabajo de campo para cada uno de los cuatro grupos se estableció de la siguiente forma:

1. Introducción al entorno virtual por parte de los investigadores.

2. Presentación de la prueba Pretest.

3. Trabajo autónomo de cuatro semanas en el entorno teniendo como eje las revistas virtuales. A la par, estuvieron habilitadas las ayudas interactivas como lo fueron: el foro, el chat $\mathrm{y}$ encuentros sincrónicos con los investigadores $\mathrm{y}$ estudiantes pasantes para aclarar dudas.

7. Presentación de la prueba postest.

8. Sesión de cierre para aplicar la encuesta de percepción.

\section{Fase 5. Análisis de datos}

Para el análisis correlacional, se tuvieron en cuenta las dos evaluaciones aplicadas en los diferentes momentos establecidos. Como ya se dijo, entre el momento del pretest y al finalizar la intervención con el postest, hubo un periodo prudente de revisión de la revista por parte de cada grupo de estudiantes que comprendió 4 semanas. En ese lapso de tiempo, tuvieron la oportunidad de mirar toda la información que tenía cada unidad entre teoría y videos, así como las actividades de gamificación que comprendían, entre otras: sopas de letras, quices, preguntas de emparejamiento y de completar, establecidos desde la parte teórica de la propuesta. Aunque esas actividades no se tuvieron en cuenta para los análisis de resultados, si permitieron verificar la interacción de los estudiantes con las revistas virtuales.

\section{RESULTADOS Y DISCUSIÓN}

Como se dijo anteriormente, para llevar a cabo esta parte se contrastaron las pruebas pretest y postest que establecieron como variables. Ese contraste se hizo a partir de varios parámetros, los cuales fueron: el promedio, la desviación estándar, el coeficiente de correlación y la prueba t-student. Cabe anotar que los dos momentos contaron con cuestionarios de aproximadamente 50 preguntas, principalmente de selección 
múltiple. El puntaje se midió con una escala de 0 a 5 , donde 5 era la nota más alta. A continuación, se presentarán cada uno de los parámetros analizados. En primera instancia, en la figura 5 se observan la comparación de los promedios de cada entorno:

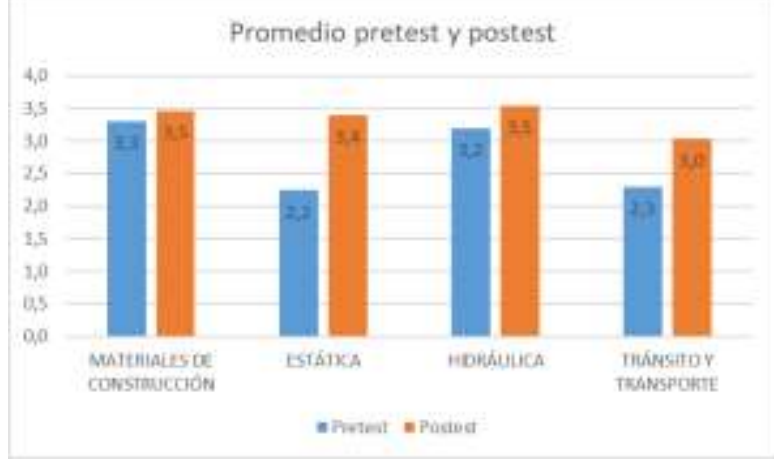

Figura 5. Comparación promedios pruebas pre y postest.

Como se observa en la figura 5, en cada revista se mejoró del postest con respecto al pretest. Otro aspecto a destacar, es que en el postest todos los resultados estuvieron por encima de 3. Particularmente, en las dos revistas en donde se obtuvieron más bajos resultados en el pretest fue en donde más se mejoró en el postest con respecto a esa prueba inicial, Estática y Tránsito y Transporte.

Enseguida se tiene la figura 6 que compara las desviaciones estándar:

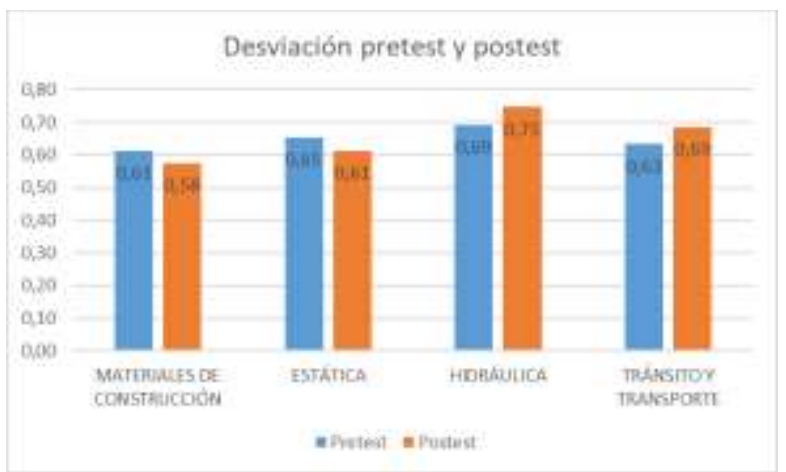
y postest

Figura 6. Comparación desviaciones estándar pruebas pre

Como se observa en la figura 6, las desviaciones estándar entre el pretest y el postest fueron muy cercanas en cada uno de los entornos. Éstas en general, fueron bajas en los dos momentos, lo cual fue algo positivo para la intervención, ya que esté parámetro destaca la homogeneidad de los grupos durante todo el proceso y no interviene negativamente para otros análisis.

Por otra parte, para realizar los análisis de correlación, se tuvo en cuenta la tabla 2 con los criterios para los mismos y que permitirán interpretar respecto a este parámetro:
Tabla 2. Criterios para el coeficiente de correlación

\begin{tabular}{|l|l|}
\hline VALOR & CRITERIO \\
\hline $\mathrm{r}=1$ & Correlación perfecta positiva \\
\hline $0.9 \leq \mathrm{r}<1$ & Correlación muy alta \\
\hline $0.7 \leq \mathrm{r}<0.9$ & Correlación alta \\
\hline $0.4 \leq \mathrm{r}<0.7$ & Correlación moderada \\
\hline $0.2 \leq \mathrm{r}<4$ & Correlación baja \\
\hline $0<\mathrm{r}<0.2$ & Correlación muy baja \\
\hline $\mathrm{r}=0$ & Correlación nula \\
\hline $\mathrm{R}=-1$ & Correlación perfecta negativa \\
\hline
\end{tabular}

Ahora, en la figura 7 se observa el coeficiente de correlación de cada entorno:

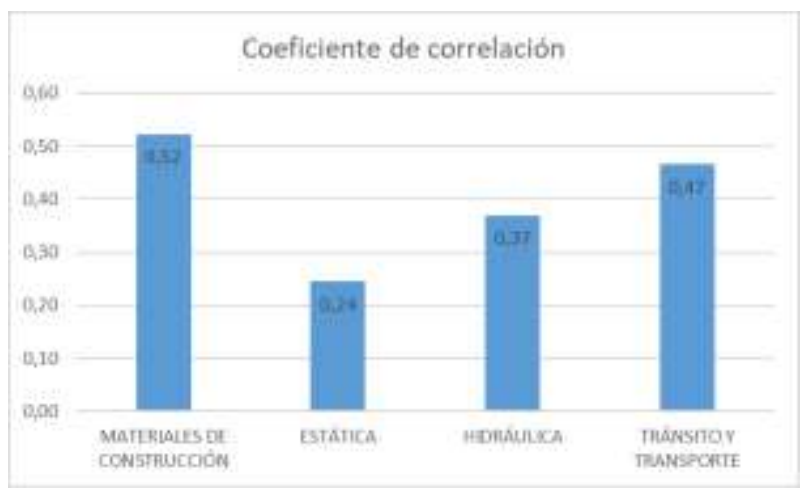

Figura 7. Comparación coeficientes de correlación.

De acuerdo con la figura 7, se observa que dos de los cuatro entornos (Materiales de Construcción y Tránsito y Transporte) tuvieron una correlación moderada, los otros dos tuvieron correlación baja (Estática e Hidráulica). En ese sentido, este parámetro permitió establecer que no hubo una asociación entre las variables pretest y postest en los cuatro entornos, por lo tanto, no se pudo tener como referente de tendencia, ya que era fiable.

Teniendo en cuenta que la correlación no fue un indicativo de análisis claro ente el antes y el después de la intervención, se analizó la significancia entre las variables con la prueba tstudent. Esta prueba permitió revisar si hubo diferencias significativas de resultados entre los dos momentos (pre y postest). Antes de mostrar la tabla de resultados, cabe recordar que el valor $\mathrm{p}$ de referencia es de 0,05 , si la prueba $t$ arrojaba un valor menor a este, se podía establecer que había diferencias significativas, si el valor $\mathrm{p}$ era mayor de 0,05 , no había diferencias significativas. En ese aspecto se establecieron las hipótesis nula y alternativa de la siguiente forma:

Hipótesis nula (Ho): no existen diferencias significativas entre los resultados de la prueba pretest y la prueba postest en los cuatro entornos virtuales apoyados por revistas digitales.

Hipótesis alternativa ( $\mathrm{Ha}$ ): los resultados de la prueba postest van a ser mejores significativamente que los resultados 
de la prueba pretest en los cuatro entornos virtuales apoyados por revistas digitales.

De acuerdo con el planteamiento de las hipótesis, en la tabla 3 se observa el valor p de cada entorno:

Tabla 3. Valor $p$ para el pretest y el postest de cada revista.

\begin{tabular}{|l|l|}
\hline ENTORNO & VALOR P \\
\hline MATERIALES DE CONSTRUCCIÓN & 0,02 \\
\hline ESTÁTICA & 0,0000000001 \\
\hline HIDRÁULICA & 0,03 \\
\hline TRÁNSITO Y TRANSPORTE & 0,000000001 \\
\hline
\end{tabular}

Como se observa en la tabla 3 , el proceso de trabajo con los cuatro entornos permitió establecer que, si hubo diferencias significativas a favor de la intervención, ya que todos los valores $\mathrm{p}$ fueron menores a 0,5 . Lo anterior quiere decir que los resultados de la prueba postest fueron mejores significativamente que los resultados de la prueba pretest en los cuatro entornos virtuales apoyados por revistas digitales. Cabe aclarar que estuvo se evidenció de mejor forma en los entornos de Estática e Hidráulica.

En términos generales, el efecto inclusivo de la intervención pedagógica se centró en el diseño de actividades digitales basadas en contenidos específicos propios de Ingeniería Civil y en la evidencia del impacto positivo de los estudiantes de este programa que se muestra en el uso del entorno virtual a través de las revistas digitales. Esta categoría resume el hecho de que, durante el proceso de implementación, las actividades (esto es, los materiales) desarrolladas tuvieron un efecto positivo en los estudiantes, que se familiarizaron con el mismo tipo de materiales en su proceso de aprendizaje: principalmente las revistas y las actividades interactivas y de gamificación en ellas, mostrado significativamente en las cuatro revistas digitales.

Durante las fases del proyecto de investigación se implementó el entorno virtual con las revistas digitales, se contrastó con diferentes momentos del desarrollo y se llegó a los siguientes resultados: se cumplió con el objetivo general de desarrollar una propuesta digital que potenciara el uso del inglés técnico aplicado al plan lector en unos cursos del programa de Ingeniería Civil mediante un entorno virtual basado en revistas interactivas propias de la disciplina y apoyadas en la metodología ESP.

Los objetivos específicos fueron logrados uno a uno durante el proceso de investigación, de la siguiente manera: se diseñaron entornos virtuales en Moodle apoyados por revistas digitales basadas en la metodología ESP que potenciara el vocabulario en inglés en los estudiantes; se implementaron diversas actividades online a partir de la gamificación haciendo uso de entornos en la plataforma Moodle y las revistas digitales; y se validó el plan lector a partir del trabajo en los entornos virtuales con el grupo de estudiantes de la facultad de Ingeniería Civil, mostrando diferencias significativas entre los resultados a favor de la intervención. En ese sentido, aunque la correlación entre el antes y el después en ninguna de los cuatro entornos fue alta, si se resalta que los promedios mejoraron en el postest respecto al pretest en los cuatro, aunque más marcado en dos de ellos (Estática y Tránsito y Transporte), más por los bajos resultados de la prueba pretest en estos dos entornos. De acuerdo a lo anterior se la hipótesis nula y se acogió la hipótesis alternativa que decía que: los resultados de la prueba postest van a ser mejores significativamente que los resultados de la prueba pretest en los cuatro entornos virtuales apoyados por revistas digitales. También se evidenció que, aunque no era un aspecto de estudio, los resultados finales en las pruebas postest de los cuatro entornos tuvieron resultados cercanos y por encima de 3 .

Por otra parte, para los estudiantes de la Licenciatura en Lenguas Modernas con Énfasis en Inglés, el diseño de este tipo de material virtual fue muy positivo para su futuro desempeño como docentes, puesto que el uso de recursos tecnológicos, principalmente, en el desarrollo de este enfoque específico (ESP) disciplinar fue enriquecedor.

Finalmente, desde los análisis también se pudo observar que, la interacción en los entornos virtuales exigió que el plan lector desde las revistas los estudiantes trabajaran en jornada extra clase, permitiendo así a los estudiantes avanzar con relación a su nivel de habilidad léxica del idioma inglés. Un aspecto a señalar es el interés tanto por parte de los investigadores como de la muestra intervenida, por continuar con el trabajo, para obtener los resultados deseados, no solo en el idioma inglés, sino potenciar sus habilidades disciplinares, haciendo de estos entornos virtuales mediado por revistas digitales un pretexto para avanzar a un proceso de apropiación de una lengua extranjera.

\section{CONCLUSIONES}

En el desarrollo de este trabajo investigativo se evidenciaron procesos de aprendizaje en varias direcciones: en la primera, se potenció la adquisición léxica de la lengua extranjera, en este caso inglés, relacionada con el plan lector, se fortalecieron contenidos disciplinares a través de la gamificación y refuerzo permanente, se aprovechó el uso de recursos tecnológicos como las herramientas online, a través del diseño de un entorno virtual y unas revistas organizadas digitalmente. posteriormente, se fortaleció la habilidad léxica específica de la disciplina de los futuros Ingenieros Civiles, su vocabulario y temática establecida previamente. 
La interpretación de diferentes tipos de textos en inglés favoreció la consecución de los objetivos propuestos, así como también el empoderamiento léxico a través de actividades de gamificación. Este resultado es efectivo en relación con lo que señalaron [2] Bonk, C. J., \& Graham, C. R. (2012), quienes manifiestan la implementación de metodologías eclécticas para el aprendizaje de una lengua extranjera por medio de entornos virtuales. El proceso llevado a cabo reforzó dicha premisa y la validó.

Asimismo, y de acuerdo a las interpretaciones de la encuesta de percepción que se aplicó al final del proceso, el uso del entorno virtual Moodle y las revistas digitales hizo efectivo el trabajo individual y el manejo del tiempo; haciendo del proceso de aprendizaje de inglés técnico, un camino lúdico y con resultados a corto y mediano plazo. Por lo tanto, el proceso desarrollado demuestra que los conceptos disciplinares y la necesidad ocupacional prima, haciendo evidente la motivación del estudiante de Ingeniería Civil en este caso.

En relación con la evaluación, los resultados se observaron en varias direcciones: un resultado positivo fue la apropiación de los diversos recursos y los procesos de auto, co y heteroevaluación incluidos en las revistas digitales, así como también la implementación de las mismas en los espacios disciplinares. Aquí [4] Hernández (2012) señala que las estrategias virtuales deben mostrar diferentes caminos para profundizar en las temáticas, procesos que se evidenciaron en la inclusión de los links.

Todo lo anterior se refuerza a partir de los resultados cuantitativos de la intervención, ya que se logró mejorar el promedio en los resultados del postest en todas las revistas. De igual forma, se encontraron diferencias significativas de mejoría en las evaluaciones postest corroborando la hipótesis alternativa. Respecto a la correlación, no fue alta en las cuatro revistas, lo que supone que este parámetro hay que indagarlo de manera más profunda en una nueva intervención, ya que no permitió una asociación entre las variables pretest y postest, lo que no dio fiabilidad en este aspecto. Las desviaciones estándar reflejaron que la dispersión no fue un parámetro que jugó en contra de la intervención, y que, por el contrario, mostró grupos homogéneos en este aspecto.

Las anteriores apreciaciones dejan ver que, independientemente del nivel de inglés que tenían los estudiantes de la muestra, la intervención permitió verificar la potenciación de dicho nivel, a partir de la mejoría en los resultados de la prueba pretest, lo que indicó una mejoría significativa en los cuatro entornos planteados y, que es lo más importante, a través de la adquisición del inglés técnico propio de cada uno de los cursos disciplinares seleccionados, lo que le da un realce importante a la metodología pedagógica seleccionada para el aprendizaje de un idioma extranjero en este caso, la cual fue ESP.
Finalmente, cabe citar aquí que en tiempos de pandemia por COVID 19, los estudiantes tuvieron la oportunidad de exponerse e interactuar con mayor facilidad que en cualquier momento presencial. La situación que se vivió en la educación y en este caso en la superior y la forma como se afrontó la estadía en casa permitió fortalecer las habilidades relacionadas con la lengua extranjera y monitorear los avances en un proceso $100 \%$ virtual.

\section{REFERENCIAS}

[1] Bhatia, V. (2008). Lenguas con Propósitos Específicos: Perspectivas cambiantes y nuevos desafíos. Scielo. Revista signos, 41(67), 157-176. Recuperado https://scielo.conicyt.cl/scielo.php?script=sci arttext\&pid=S071809342008000200006\&lng=en\&nrm=iso\&tlng=en

[2] Bonk, C. J., \& Graham, C. R. (2012), Handbook of blended learning: Global Perspectives, local designs. San Francisco, CA: Pfeiffer Publishing

[3] Bueno, C. y Hernández, M. (). Inglés con fines específicos: entonces y ahora. English for Specific Purposes: A historical perspective. Instituto Superior de Ciencias Médicas "Carlos J. Finlay"

[4] Hernández Sampieri, R. Metodología de la investigación. Distrito Federal, México: McGraw Hill, 2010.

[5] Lee, L. (2016). Autonomous learning through task-based instruction in fully online language courses. Language Learning \& Technology, 20(2), 81-97. Tomado de: http://1lt.msu.edu/issues/june2016/lee.pdf

[6] McDougald, J. (2014). AICLE: Un nuevo enfoque para el aprendizaje bilingüe. Artículo central nacional. Ruta Maestra ed. 11

[7] Murray G, Lamb T y Xuesong G. (2011), Identity, Motivation, and Autonomy in Language Learning. Libro, P. 177

[8] Nadera Boukhatem (2015), Promoting student motivation in EFL classroom: Through extended music education. Published by Elsevier Ltd. 368-371. Tomado de

[9] Thomas, M., \& Reinders, H. (Eds.). (2010). Task-based language learning and teaching with technology. A\&C Black.

[10] Vidal, K. (2005). El inglés para fines específicos y la universidad: un estudio empírico exploratorio. Volumen monográfico 213-231. 